therapy) in 2,166 stable high-risk patients who had persistent total occlusion of the infarctrelated coronary artery 3-28 days after myocardial infarction. The primary end point was defined as a composite of death, myocardial reinfarction and NYHA class IV heart failure. The group treated with $\mathrm{PCl}$ plus optimum medical therapy had a 4-year cumulative primary event rate of $17.2 \%$, compared with $15.6 \%$ for the group receiving optimum medical intervention only (hazard ratio $[H R] 1.16 ; P=0.20$ ). The number of deaths $(9.1 \%$ vs $9.4 \%$; HR $1.03 ; P=0.83$ ) and frequency of class IV heart failure (4.4\% vs $4.5 \%$; HR 0.98; $P=0.92)$ were similar in the two groups. There was, however, a trend towards higher rates of reinfarction in the group that received $\mathrm{PCl}$ than in the group that received medical therapy alone $(7.0 \%$ vs 5.3\%; HR 1.36; $P=0.13$ ).

$\mathrm{PCl}$ after the currently accepted reperfusion period did not offer any benefit to patients in terms of reducing rates of death, reinfarction, or heart failure. Instead, the authors conclude that $\mathrm{PCl}$ carried out 3-28 days after myocardial infarction might be associated with higher rates of reinfarction.

Original article Hochman JS et al. (2006) Coronary intervention for persistent occlusion after myocardial infarction. N Engl J Med 355: 2395-2407

\section{Multipotent embryonic cardiac progenitor cells implicated in cardiogenesis}

There are two distinct populations of cardiac progenitor cells; one of these populations, which contributes to the formation of the outflow tract, right ventricle, portions of the left ventricle and the atria, is characterized by the expression of the transcription factor islet-1 (is/1). Whether different cardiovascular cell types arise from distinct precursor cells or from a single lineage of multipotent stem cells has been unclear. Moretti et al. have now identified a subset of multipotent is/1+ cardiovascular progenitor (MICP) cells that can give rise to cardiac muscle, smooth muscle or endothelial cells during embryonic heart development in mice.

Investigations showed that MICP cells are defined by the transcriptional signature is $/ 1^{+} / \mathrm{Nk} \times 2.5^{+} / \mathrm{flk} 1^{+}$. Single-cell-derived clones of MICPs can spontaneously develop into cardiac, smooth muscle or endothelial cells. Cells that retained expression of is 11 and $f l k 1$ gave rise to endothelial or smooth muscle cells, whereas cells that retained Nkx2.5 expression (with or without is/1) developed into cardiac or smooth muscle cells. The authors suggest that differential expression of the three genes leads to the development of distinct populations of restricted progenitor cells; for example, is $/ 1^{+} / f l k 1^{+}$cells might represent a population of vascular progenitor cells, while $N k \times 2.5^{+}$cells might give rise to muscle.

The authors conclude that clonally derived heart progenitor cells have potential for therapeutic use in the regeneration of damaged heart tissues, as these cells offer the possibility of matching the cell expression profile to a specific cardiac structure. Furthermore, the restricted differentiation potential of these cells would have safety advantages compared with multipotent embryonic stem cells.

Original article Moretti A et al. (2006) Multipotent embryonic isl $1^{+}$progenitor cells lead to cardiac, smooth muscle, and endothelial cell diversification. Cell 127: 1151-1165

\section{Volumetric optical microscopy prototype shows promise for optical biopsy in vivo}

Wide-field optical biopsy could enable the identification of vulnerable plaques in vivo before rupture. Now, Yun et al. have developed a minimally invasive fiberoptic imaging technique-optical frequency-domain imaging (OFDI) - that can access remote locations within the body and rapidly capture high-resolution, cross-sectional images.

Intravascular optical imaging is complex because blood cells scatter light. Although displacing blood with saline can circumvent this problem-providing a few seconds of clear viewing - to date optical imaging has been limited to point sampling, as the sampling rate of conventional optical coherence tomography is slow. Using a prototype system, the researchers carried out a comprehensive microscopic study of porcine coronary arteries. With the improved image acquisition rate afforded by OFDI, long sections of the three main coronary arteries in five living swine were imaged. In total, 56 volumetric 